\title{
ZUR QUARTÄRGEOLOGIE IM GRENZBEREICH ZWISCHEN MUOTA/REUSS- UND LINTH/RHEINSYSTEM
}

\author{
René HantKe
}

PROBLEMSTELLUNG

Mit talgeschichtlichen Problemen im Grenzgebiet zwischen Reuß- und Linthgletscher beschäftigten sich schon zahlreiche Forscher, so besonders Alb. Heim (1894a, b, 1919), A. Aeppi.I (1894, 1904), O. FreY (1907), J. FrüH (1907), E. BRÜcKNER (1909), RoMAN Frei (1912, 1914) und in neuerer Zeit vor allem J. Kopp (1937, 1961a, b), H. Jäckli (1956) und H. SUTER (1956, 1960).

Besonders bewegt gestaltete sich die Erforschungsgeschichte um das pleistozäne Geschehen, insbesondere um die verschiedenen Schottervorkommen und deren Altersdeutung, im zugerischzürcherischen Grenzgebiet, zwischen Wädenswil und Zug und zwischen dem Zugersee und dem Knonauer Amt.

Im Abschnitt Wädenswil-Zug standen sich namentlich die beiden Auffassungen ALb. HeIMS und AEPPLIS einerseits und E. BrücKNers und R. Freis andererseits scharf gegenüber. Während die Schotter an der Sihl und an der Lorze von Alb. Heim und Aeppli mit den Schottern des Albiskammes in Verbindung gebracht und als bei den letzten alpinen Bewegungen rückgesenkte Deckungsschotter der Günz-Eiszeit zugewiesen wurden, ordnete sie FrEI auf Grund minutiöser Untersuchungen zum Teil der Hochterrassen-Eiszeit, zum Teil der größten Vergletscherung zu. BRücKNER (1909:510) betrachtete die tiefsten Schotter an der Sihl und an der Lorze sogar als jungglazial, als Schotter der präwürmeiszeitlichen Laufenschwankung PENCKs, diejenigen des höheren Niveaus als solche der Riß-Eiszeit.

Auch hinsichtlich der Schotter des nördlich anschließenden Knonauer Amtes scheinen die Meinungen auseinander zu gehen, so daß ihre Altersdeutung ebenfalls zu erneuten Überlegungen anregen mußte.

\section{DIE TALUNG DES LAUERZER- UND DES ZUGERSEES}

Zwischen Goldau und Oberarth konnte J. Kopp (1937) einen durch prähistorische Bergsturzmassen eingedeckten Schluchtabschnitt feststellen. Neulich versuchte dieser Autor (1961a) die eingedeckte Rinne als alten Muotalauf zu interpretieren. Durch den prähistorischen Bergsturz von Oberarth wäre die Muota zwischen dem Talkessel von Schwyz und dem Zugerseebecken gestaut worden, so daß sich hinter dem Bergsturzriegel der Lauerzersee aufgestaut hätte.

Eine solche Deutung des Schluchtabschnittes von Goldau-Oberarth läßt aber eine Reihe von weiteren, morphologisch bedeutsamen Tatsachen außer Acht. So fehlen für einen derartigen alten Muotalauf im Talkessel von Schwyz, zwischen Muotaschlucht am Ausgang des Muotatales und dem flachen Sattel von Goldau, jegliche weiteren Anhaltspunkte.

Die Talung zwischen Seewen und dem Knie des Vierwaldstättersees bei Brunnen, die heute von der Seeweren, dem Abfluß des Lauerzersees, und von der bei Ibach in die Ebene ausbrechenden Muota durchflossen wird, ist in ihrer Anlage tektonischer Natur. Sie entstand als Furche zwischen dem Stirngewölbe des nördlichsten Teilelementes der Drusberg-Decke, dem Axensteingewölbe, und den bei deren Vormarsch an der Basis mitgeschleppten und bis an den Alpenrand verfrachteten Kreide/Eozän-Platten des Urmiberges und der Rigi-Hochflue. Die Furche selbst ist lediglich von wenig resistenten Gesteinen südhelvetischer Oberkreide- und Alttertiär-Schuppen erfüllt. Wie bereits früher ausführlich dargelegt wurde (R. HANTkE, 1961a), endet die Schichtplatte des Urmiberges bei Seewen an einer Querstörung. Ihre östl. Fortsetzung ist weder in der Tiefe zu suchen, noch wurde sie aberodiert. Die Kreideserie des Urmiberges setzt vielmehr in der Chaiserstock-Kette ebenso unvermittelt wieder ein wie sie bei Seewen endet. Vom Chaiserstock läßt sie sich über Wasserberg-Silberen bis ins Roßmattertal westlich des Glärnisch verfolgen, wo sie abermals an einer Querstörung ab- 


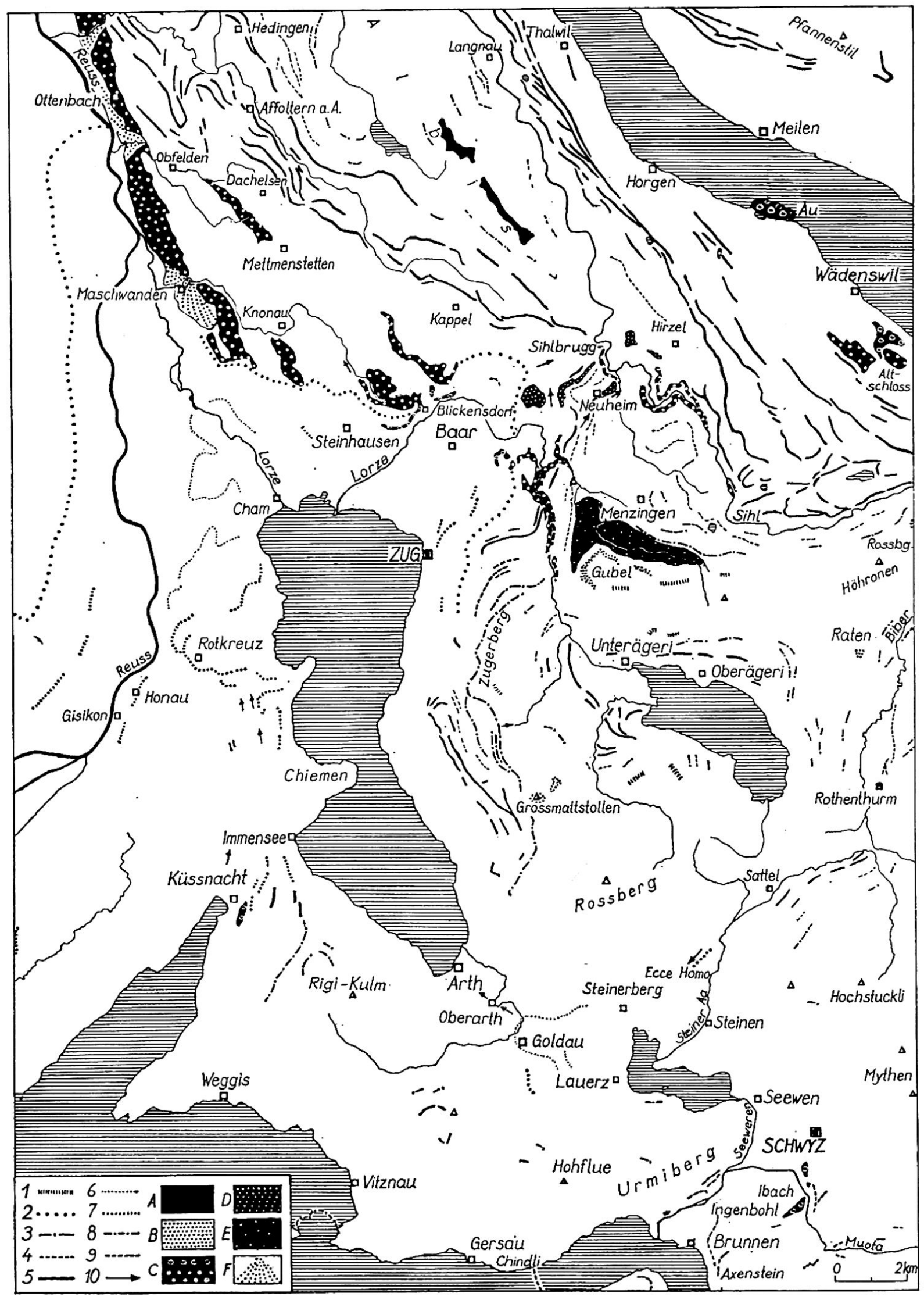

Fig. 1. Wallmoränen, Eisstände und Schotter zwischen Reuß- und Zürichseetal. 1 Spätriß-Wallmoränen, 2 Frühwürm-Eisrandlage, 3-5 Hochwürm-Moränen: 3 Killwangen/Mellingen-, 4 Schlieren/ Stetten-, 5 Zürich/Bremgarten-Stadium, 6-9 Spätwürm-Moränen: 6 und 7 Hurden/Gisikon-, 8 Vitznau/Goldau-, 9 Chindli/Seewen-Ingenbohl-Stadium, 10 Abflußrinnen. A Älterer Deckenschotter $(?=\mathrm{B}), \mathrm{E}$ Spätriß-Vorstoßschotter, C tiefere bzw. höhere Frühwürmschotter, D höchste Frühwürmschotter, E Hochwürmschotter, F Spätwürm-Deltaschotter. 
reißt, an der Basis der höheren Decken an den Alpenrand geschleppt wurde und in den Aubrigen wieder erscheint.

Bei Seewen stellte sich damit seit dem Aufbranden der Urmiberg-Platte und des subalpinen Flysches auf der starren Rigi-Nagelfluh eine tiefe Bresche ein.

Leider sind wir über die präwürmeiszeitliche Überprägung dieses Abschnittes sehr schlecht unterrichtet, so daß wir über alte Flußläufe, insbesondere über riß/würm-interglaziale Abflußrinnen, nichts Sicheres aussagen können. Hingegen hinterließ die Würm-Eiszeit, namentlich ihre Schlußphasen, deutlichere Indizien.

Als der Reußgletscher zur ausgehenden Würm-Eiszeit im Vierwaldstättersee nördlich der Nasen die unterseeische Stirnmoräne von Vitznau aufschüttete, war auch der Talkessel von Schwyz noch von Eis erfüllt. Damals teilte sich der Reußgletscher bei Brunnen; während sich der eine Arm zwischen Seelisberg und Hochfluekette gegen W wandte, vereinigte sich der andere Arm am Ausgang der Muotaschlucht mit dem Muotagletscher und stieß über Seewen noch bis gegen Goldau vor, was auf der SW-Seite des Lauerzersees durch tiefgelegene Wallreste und auf der Nordseite des Sees durch zahlreiche Reußerratiker eindrücklich dokumentiert wird. Der Lauerzersee ist demnach nicht als Bergsturzsee, sondern als Zungenbecken eines Gletscherlappens aufzufassen (cf. R. Hantke 1958). Diese Interpretation wird auch durch den flachen Seeboden und die rückläufige Entwässerung gestützt.

Die von J. Kopp nachgewiesene Schlucht zwischen Goldau und Oberarth wäre demnach nicht als Rest eines alten Muotalaufes, sondern vielmehr als alte Schmelzwasserrinne $\mathrm{zu}$ deuten, welche während des Vitznau/Goldau-Stadiums funktionierte.

$\mathrm{Ob}$ die Muota je einmal während einer Interglazialzeit über Seewen-Goldau durch die Zugersee-Talung abfloß, läßt sich heute noch nicht sicher entscheiden. Auf jeden Fall existierte neben dem Durchbruch von Seewen auch die Talung IbachBrunnen schon sehr früh.

Ganz analog wie der Schmelzwasser-Abfluß von Oberarth sich zeitlich einstufen läßt, so dürfte noch eine zweite von J. Kopp (1961a) dargelegte Beobachtung, der alte Lauf der Steiner Aa von Ecce Homo durch die Alluvialebene von Talacher Richtung Lauerzersee, mit einem Rückzugstadium des Muota/Reußgletschers in Zusammenhang $\mathrm{zu}$ bringen sein. Ein solcher einstiger Lauf der Steiner Aa würde gegen Sattel einen Eisrand des Muota/Reußgletschers voraussetzen, der dem Gisikon-Stadium (= Hurden-Stadium des Linth/Rheingletschers) gleichkäme, dessen Verlauf des Eisrandes im Gebiet des Zugersees auf Fig. 1 dargestellt wurde.

Ob die Sande und Schotter, die Kopp (1945, 1961a, b) in einer Bohrung bei Dörfli E von Immensee antraf, tatsächlich als Füllung eines alten Reußlaufes interpretiert werden dürfen, ist aus einer einzigen Bohrung allein schwer zu entscheiden. Möglicherweise sind diese mit den Schieferkohlen führenden Schottern von Küßnacht am Rigi in Zusammenhang zu bringen, die sehr wahrscheinlich als frühwürmeiszeitlich zu deuten sind und die beim Vorstoß der würmeiszeitlichen Gletscher im Winkel zwischen dem Küßnachterarm und dem Zugerseearm des Reußgletschers zur Ablagerung gelangten. Ihr Alter wird aber noch durch eine $\mathrm{C}^{14}$-Analyse zu überprüfen sein.

Daß auch später, zur Zeit des spätwürmeiszeitlichen Stadiums von Gisikon, als der Küßnachterarm des Vierwaldstättersees und der Zugersee noch von Eis erfüllt waren (cf. Fig. 1), Schmelzwässer außerhalb der Endmoränen zwischen Küßnacht, der Halbinsel Chiemen und Rotkreuz längs vorgezeichneten Molassestörungen Abflußwege suchten, ergibt sich aus der Detailmodellierung dieses Abschnittes (cf. LK 1131 und $1151)$.

\section{DIE $\ddot{A G E R I-T A L U N G}$}

Zur Zeit, als der vorstoßende frühwürmeiszeitliche Muota/Reußgletscher die Ebene von Baar erfüllte, war ein seitlicher Eisarm von Steinen über den Sattel in die Talung 
des Ägerisees eingebrochen. Wie sich aus der Oberflächengestalt ergibt, vor allem aus den etwas außerhalb des heutigen Seebeckens gelegenen markanten Endmoränen - sie entsprechen dem hochwürmeiszeitlichen Zürich-Stadium - dürfte die Gletscherstirn damals etwa bei Unterägeri gelegen haben, so daß das Becken des Ägerisees noch vollständig von Eis erfüllt war.

Durch ein bereits während der ausgehenden Riß-Eiszeit kräftig eingetieftes altes Lorzental ergoß sich beim Vorstoß des würmeiszeitlichen Gletschers, zusammen mit den Schmelzwässern, ein mächtiger Schotterstrom: die Lorzenschotter, welche schon von R. FREI (1912) sehr eingehend untersucht worden sind.

In der Brüggli-Risi im Lorzentobel liegen über diesen Schottern konkordant und ohne Verwitterungsschicht mächtige jüngere Moränen- und Schottermassen. FREI (1912: 60) schloß daraus richtig, «der Unterbruch in der Akkumulation zwischen dem älteren Schotter und den jüngeren Moränen sei nur von sehr kurzer Dauer gewesen ...». Merkwürdigerweise stellte er die Lorzenschotter dennoch in die durch eine Interglazialzeit getrennte größte Vergletscherung.

Daß die Ägeri-Talung nicht erst in der Würm-Eiszeit sondern schon in einer früheren Eiszeit von einem analog verlaufenden Muota/Reußgletscher durchströmt worden war, wird durch einen von FREI $(1912: 43,60)$ in den Lorzenschottern festgestellten Block eines verfestigten älteren Schotters dokumentiert. Als älteres Schottersystem fällt im Lorzentobel nur der Gubelschotter in Betracht, der altersmäßig, wie noch dargelegt werden wird (S.218), am ehesten als spätrißeiszeitlich zu datieren ist.

Aus der Gegend des Ägerisees erwähnt FREI (1912: 73/74, 1914:6) hochgelegene Schottervorkommen an der Brandflue und von der Gipfelkuppe des Großmattstollen (3,5 bzw. $4 \mathrm{~km}$ SSW Unterägeri). Da sie rund $100 \mathrm{~m}$ über den höchsten würmeiszeitlichen Moränen (cf. R. HavtKe 1958) liegen, dürften sie ebenfalls dem Spätriß zugeordnet werden.

Auch SE des Raten, dem Paßübergang zwischen Oberägeri und Biberbrugg, fand R. FREI stark verfestigte Schotter mit gekritzten Geröllen. Da diese Verrucano-Gerölle führen, muß der Linth/Rheingletscher im Spätriß noch kräftig in die von der Biber entwässerte Talung vorgestoßen sein und dabei den über Rothenthurm vordringenden Muota/Reußgletscher am weiteren Vorstoß gegen Biberbrugg gehindert haben.

\section{DIE ALTEN TALUNGEN ZWISCHEN DEM OBEREN ZÜRICHSEE UND DER EBENE VON BAAR}

Von hoher Bedeutung für die geologische Geschichte des Abschnittes zwischen dem Oberen Zürichsee, dem Zugersee und dem Knonauer Amt ist die Altersdeutung der verschiedenen, sich durch starke Verkittung auszeichnenden Schotter.

Die Verkittung dieser Schotter ist sehr wechselvoll. Auffallend stark verkittet sind vor allem diejenigen in der Gegend des Sihlsprungs. Doch erwähnten schon A. AEPPLI, E. BRüCKNER und R. FREI in diesen Schottern auch sehr lockere Partien.

Alb. Heim $(1894,1919)$ deutete all die tiefliegenden, stark verkitteten Schotter zwischen Zürichsee und Zugersee als Erosionsrelikte einer früher mit den Deckenschottern auf dem Albiskamm zusammenhängenden, gegen die Alpen hin kontinuierlich ansteigenden Platte. Ihre tiefe Lage in der westlichen Fortsetzung seiner rückläufigen Erosionsterrassen am Oberen Zürichsee erklärte Heım, wie diese selbst, als Folge einer späten, jungquartären Rücksenkung des Alpenkörpers, die in diesem Gebiet $425 \mathrm{~m}$ betragen würde.

Vom Deckenschotter der Albiskette, welcher der Günz-Eiszeit zugeordnet wird, unterscheiden sich die Schotter zwischen Zürichsee und Zugersee schon im Geröllinhalt. R. FREI (1912) hält dabei zwei Schottersysteme auseinander: ein höheres, welches die Vorkommen von Ober Kellenholz (= Chälen LK), Josefsgütsch bei Neuheim und der Baarburg umfaßt, und ein tieferes System, dasjenige der eigentlichen Sihl- und Lorzenschotter. Während FREI die höheren Schotter der drittletzten Vergletscherung, 
der Hochterrasse - im Text allerdings mit Vorbehalt - zuwies, ordnete er die tieferen Schotter der größten Vergletscherung zu.

Hinsichtlich der Lagerungsverhältnisse konnte FREI übereinstimmend mit BRÜcKNER (1909) feststellen, daß sich nirgends Anzeichen einer Rücksenkung beobachten lassen.

Die Altersfrage der verkitteten Schotter bei Altschloß, zwischen Richterswil und Wädenswil, und beim Waisenhaus Wädenswil ließ FrEI (1912:64) offen, da kein sichtbarer Zusammenhang mit einem der beiden Schottersysteme im Sihl- und Lorzental besteht. Lagemäßig entsprechen diese beiden Vorkommen jedoch tatsächlich, was auch schon R. FrEI festgestellt hatte, den tieferen Schottern, denjenigen des Sihlsprungs.

Die etwas tiefer gelegenen Eisrand-Stauschotter im Reitbachtal SE von Wädenswil und die Schotter der Halbinsel Au dürften einen etwas früheren, noch weniger hoch hinaufreichendem Eisstand des Linth/Rheingletschers bekunden. Von A. AepPLI (1894) wurden die Schotter von Altschloß und beim Waisenhaus Wädenswil noch als Deckenschotter, diejenigen im Reitbachtal als Niederterrassenschotter bezeichnet.

E. BRÜCKNER wies die Schotter an der Sihl und an der Lorze der Würm-Eiszeit zu. Er betrachtete diese als Reste einer großen, nachträglich mit Moräne überschütteten, schwach gegen NW abfallenden Schotterflur, welche im Sihltal die höher gelegenen, von ihm ebenfalls als rißeiszeitlich aufgefaßten Schotter durchbrach.

$\mathrm{Da}$ die Schotter verschiedentlich deutlich auf Moräne aufliegen, was auch ihren außerordentlichen Wasserreichtum verständlich erscheinen läßt, in den untersten Partien mit Moräne wechsellagern und schließlich von Moräne überdeckt werden, stellt sie E. BRÜCKNER in die spätwürmeiszeitliche Laufenschwankung.

Wie Untersuchungen in den verschiedensten Gebieten der Alpen ergeben haben, ist nun aber die von PENCK und BRÜCKNER als Laufenschwankung interpretierte Erscheinung in der Chronologie der Würm-Eiszeit nicht nach dem Hochglazial, sondern in die Vorrückungsphase, ins Frühwürm, einzustufen.

Die hohe Lage der Sihl- und Lorzenschotter gegenüber den Haupttälern, dem $Z$ ürichseetal und der Ebene von Baar-Zug, führte BRÜCKNER darauf zurück, daß im Osten der Linthgletscher, im Westen der Reußgletscher ein seitliches Abfließen der Schmelzwässer hemmten. Der Abfluß konnte daher nur durch die tektonisch vorgezeichnete Senke zwischen den beiden Haupttalungen des Reuß- und des Linthsystems, durch die Wädenswil-Synklinale, erfolgen. Der geringe Anteil an Verrucano-Geröllen gegenüber den darüber liegenden Moränen erklärte BRÜCKNER mit einem «kleineren Gletscherstand».

Dieser «kleinere Gletscherstand» entspricht nun aber nicht einem jüngeren Gletscherrückgang, sondern einem älteren Vorstoß. Darnach hätte im Jungquartär ein erheblicher Anteil an Geröllen der Sihl- und der Lorzenschotter gar keinen größeren Ferntransport erlitten. Unter dem feucht-kühlen Klima, das den Vorstoß der würmeiszeitlichen Gletscher weitgehend bewirkt hatte, wurde die Gesteinsoberfläche durch Frosteinwirkung weitgehend aufgelockert und der dadurch entstandene Schutt durch Abschwemmung, Solifluktion und durch Schmelzwässer fortgeführt und in gefällsarmen Abschnitten wieder sedimentiert. Dabei wurden namentlich Gerölle aus der näheren Umgebung durch den linksseitigen Schmelzwasserstrang des Linth/Rheingletschers verfrachtet, insbesondere solche aus der subalpinen Molasse, aus der kalkarmen und daher wenig verkitteten Höhronen-Schüttung, sowie solche aus den verschiedenen Flyschserien, welche durch die Schmelzwässer des Sihl- und des Wägitaler Gletschers zugeführt wurden. Gerölle aus den helvetischen Kalkalpen, etwa von der linken Talseite des Glarnerlandes, treten mengenmäßig stark zurück. Solche dürften vor allem aus aufgearbeiteten rißeiszeitlichen Moränen stammen. Da die Gerölle meist kaum 
verwittert sind, dürften sie nicht längere Zeit der Verwitterung ausgesetzt gewesen sein, sondern wurden offenbar rasch eingebettet.

Der Einwand, daß sich bei Annahme eines frühwürmeiszeitlichen Alters der Sihlschotter mit einem erheblichen Anteil an Geröllen aus der Nagelfluh des Höhronen, auch Molassesandsteine in den Schottern finden müßten, ist zurückzuweisen. Die Verkittung der Höhronen-Nagelfluh ist meist derart gering, daß sich die Gerölle vielfach ohne Hammer aus den weichen, nur schwach verfestigten Sandsteinen gewinnen lassen. Die hinter etwas stärker verkitteten Gesteinsbänken der leicht S-fallenden Schichten sich bildenden Bachtreppen sind denn auch häufig erfüllt von sauber gewaschenen Geröllen, unter denen sich kaum je solche von Molassesandsteinen finden. Vielmehr wurden Molassesandsteine schon nach allerkürzestem Transport vollständig zu Sand zerrieben und fortgeschwemmt.

Während Gerölle von granitischem Sandstein in den Schottern fehlen, nimmt dessen Abreibprodukt, mit Calcit sekundär zementierter granitischer Sand, einen erheblichen Anteil ein.

Wie schon aus den Geröllzählungen von R. FreI (1912, Taf. 1, No. 105-123) hervorgeht, weicht der Geröllinhalt der Sihlschotter von der mittleren Zusammensetzung der Würm-Moränen des Linth/Rheingletschers deutlich ab. Auffällig ist vor allem der hohe Anteil an Kristallin-Geröllen sowie das Fehlen von Dolomiten, was sich neulich bei einer von Studenten des Geologischen Institutes unter Prof. R. TRümpys Leitung durchgeführten Zählung gezeigt hat.

Ein Vergleich mit Geröllen aus der Höhronen-Nagelfluh ergab übereinstimmende Kristallin-Typen. Ein Teil der Sandsteine scheint aus dem Flysch zu stammen. Ebenso konnte Einsiedler Nummulitenkalk festgestellt werden.

Wie aus neuesten geophysikalischen Untersuchungen - negative BougueR'sche Schwereanomalien - hervorgeht, kommt einer auf Grund geologischer Daten postulierten Entwässerungsrinne vom Oberen Zürichsee gegen die Ebene von Baar und weiter gegen das Knonauer Amt auch geophysikalisch eine durchaus reale Bedeutung zu (cf. N. Pavoni in F. Gassmann 1961). Die Rinne ist in ihrer Anlage mindestens rißeiszeitlich. Eine merkliche interglaziale Eintiefung ist kaum anzunehmen, da in diesem Zeitpunkt sowohl die Zürichsee-Talung als auch diejenige des Zugersees bereits als tiefe Wannen existierten, was durch interglaziale Seewannen dokumentiert wird ( $R$. Hantke 1959 a, b), so daß die beiden Talungen schon damals als Hauptentwässerungsrinnen funktionierten und nur sehr geringe Wassermassen durch die alte Querrinne abflossen.

Es stellt sich nun noch die Frage, ob die höheren Schottervorkommen, diejenigen der Baarburg, des Josefsgütschs bei Neuheim und von Chälen tatsächlich, wie dies BRÜCKNER und FREI getan haben, effektiv als Hochterrassenschotter der Riß-Eiszeit angehören, oder ob sie nicht allenfalls einem späteren frühwürmeiszeitlichen Vorrückstadium zuzuordnen sind, einem Stadium, bei dem der Linth/Rheingletscher bereits etwas über Zürich hinaus vorstieß.

Die Schottervorkommen der Baarburg, des Josefsgütschs und von Chälen können nur Stauschotter darstellen, die bei einem höheren Gletscherstand als die Sihl- und Lorzenschotter geschüttet wurden. Zufolge ihrer Lage zu den beiden Haupttalsystemen und auf Grund ihrer flachen Lagerung sowie dem seltenen Auftreten von gekritzten Geröllen können sie nur Vorstoß-Schotter darstellen. Auffällig ist ferner, daß die Schotter des Josefsgütschs und der Baarburg durchweg auf Molasse aufruhen, was auf eine vorgängige erosive Phase hindeutet. Während die Auflagerungsfläche auf Molasse, wie schon R. FrEI (1912: 33) feststellen konnte, bei der Baarburg gegen NW geneigt ist, stellt sich am Josefsgütsch ein Gefälle gegen NE ein. Moräneneinschaltungen fehlen durchweg. 
Hinsichtlich ihres Geröllinhaltes fällt wiederum, wie bei den Sihlschottern, der hohe Kristallin-Anteil auf. Ebenso stimmen die drei Analysen von der Baarburg, vom Josefgütsch und von Chälen (Ober Kellenholz) sehr gut mit denjenigen des Sihlsprungs überein (FrEI, 1912, Analysen 114, 115, 116 und 123).

$\mathrm{Da}$ die Schotter von Baarburg und von Chälen von gering mächtiger Grundmoräne überdeckt sind und diejenigen des Josefgütschs von deutlichen Seitenmoränen gekrönt werden - sie dürften sehr wahrscheinlich das Stetten (Schlieren)-Stadium dokumentieren - sind diese höheren Schotter sicher prähochwürmeiszeitlich.

Auf Grund ihrer Höhenlage dürften sie zur Ablagerung gelangt sein als der Linth/ Rheingletscher etwa bis Altstetten vorrückte und die Sihl als seitlicher Schmelzwasserstrang in Zürich durch die alte, heute von den Utomergeln und von Moränen eingedeckte prähochwürmeiszeitliche Rinne floß, welche bei Schlieren ins Limmattal einmündet.

Auffallend ist der Umstand, daß sich auch anderswo wenig außerhalb eines etwa dem hochwürmeiszeitlichen $Z$ ürich-Stadium entsprechenden Eisstand verschiedentlich Schotterfluren einstellen, die von Wallmoränen gekrönt werden: im Zürichseetal die Wulpschotter im Küsnachter Tobel, im Glattal die Schotterböden in den zur Töß entwässernden Quertälern (cf. R. HantKe 1960).

Altersgleich mit den Schottern der Baarburg, des Josefsgütschs und von Chälen dürfte sehr wahrscheinlich auch das von A. AEPPLI (1894) als Niederterrassenschotter aufgefaßte Vorkommen von Meinradsbrunnen zwischen Schindellegi und Biberbrugg sein, dem ebenfalls ein deutlicher, von der Südostbahn und der Kantonsstraße angeschnittener Moränenwall aufgesetzt ist.

Analoge Schotterfluren konnten kürzlich in der Nordostschweiz als frühwürmeiszeitlich datiert werden (cf. R. HantKe 1961 b). Auch H. Andresen (1961) konnte bei seinen Untersuchungen im Hörnlibergland verschiedentlich derartige, etwas außerhalb des Zürich-Stadiums gelegene und von Moräne bedeckte Schotter feststellen.

Solche Schottervorkommen wurden früher ausnahmslos als Hochterrassenschotter bezeichnet und der Riß-Eiszeit zugeordnet. Diese Zuordnung beruhte offenbar auf der streng dem Penck'schen Schema von den ineinandergeschachtelten Talsystemen folgenden Auffassung, wonach vor der Riß-Eiszeit durchweg alte, weite Talböden existierten, die dann während der Riß-Eiszeit nur in ihrem zentralen Abschnitt durch die Gletscher eingetieft worden wären. An den Rändern dagegen wäre noch der frühere Talboden als Schotterstreifen erhalten geblieben.

Als sicher rißeiszeitliche Schotter, als echte Hochterrassenschotter, sind zwischen Zürichsee und Zugersee, in Übereinstimmung mit FREI (1912: 64), diejenigen unter dem Kloster Gubel SSW Menzingen zu betrachten (cf. S. 215). Wie in der WürmEiszeit das Schotterfeld von Menzingen-Schurtannen im Winkel zwischen dem Ägeriarm des Muota/Reußgletschers und dem Linth/Rheingletscher, so wurden zur RißEiszeit in durchaus analoger Weise, in einem höheren Niveau, im Winkel zwischen den beiden Eisströmen Stauschotter abgelagert.

Sehr wahrscheinlich sind auch die von W. Leupold (in N. Pavoni 1955: 127) entdeckten und als Deckenschotter aufgefaßten Schottervorkommen vom Roßberg W Schindellegi als rißeiszeitliche, eine Schmelzwasserrinne füllende Schotter zu deuten.

Wohl wäre es zunächst denkbar, daß die Schotter der Baarburg, des Josefsgütschs und von Chälen allenfalls rißeiszeitliche Vorstoßschotter, also doch echte Hochterrassenschotter darstellen würden und daß dieser Schotterstrang bereits zur Riß-Eiszeit und besonders durch die Schmelzwässer des Spätriß tiefgreifend zerschnitten worden wären, wie sich dies E. BRücKNER und R. FrEI vorstellten. Dann hätten wir jedoch all diese Schotterfluren, welche einen etwas unterhalb des hochwürmeiszeitlichen $Z \ddot{u}$ rich-Stadiums liegenden Eisstand dokumentieren, einem früh- oder wahrscheinlicher 
einem spätrißeiszeitlichen Vorstoß-Stadium zuzuordnen. Bei einer solchen Altersdeutung müßten dann vor allem auch für das spätere talgeschichtliche Geschehen weitere Dokumente vorhanden sein. Insbesondere fehlt jeder sichere Beweis für eine rißeiszeitliche Zerschneidung der Schotter für Chälen - Josefsgütsch - Baarburg. Dagegen läßt sich bei Sihlbrugg beobachten, wie die höchsten Sihlschotter nach oben allmählich in die tiefsten Schotter von Chälen und des Josefsgütsch übergehen. Somit ist es viel wahrscheinlicher, daß die Zerschneidung der Schotterflur erst im Hochwürm erfolgte, diejenige $z$ wischen Josefsgütsch und Baarburg durch einen direkt nach Norden vorstoßenden Ägeriarm des Muota/Reußgletschers und dessen spätere Schmelzwässer, durch die hochwürmeiszeitliche Lorze, bot doch dieser Durchbruch den Schmelzwässern des durch das Ägerital vorstoßenden Muota/Reußgletschers die einzige Möglichkeit einer Entwässerung, diejenige zwischen Chälen und Josefsgütsch durch die linksseitigen Schmelzwässer des Linth/Rheingletschers, durch die hochwürmeiszeitliche Sihl. Von Südwesten stieß der durch die Zugersee-Talung abfließende Arm nördlich des $\mathrm{Zu}$ gerberges mächtig gegen Osten vor und dürfte wohl ebenfalls zur Hochwürm-Eiszeit entlang dem nördlichen Schotterrand die heute von der Kantonsstraße benutzte 'Talung Baar-Sihlbrugg geschaffen haben.

Wie aus dem Verlauf des Bremgarten (Zürich)-Stadiums des Muota-Reußgletschers, besonders aus dem dieses Stadium dokumentierenden Moränenwall von Hirzwangen (4 km NE Baar) hervorgeht, erfolgte zur Zeit des hochwürmeiszeitlichen $Z$ ürich-Stadiums ein Schmelzwasserabfluß in umgekehrter Richtung, von der Ebene von Baar Richtung Sihlbrugg. Aus dem Verlauf der Abflußrinnen zu beiden Seiten des Schotterstranges Chälen-Baarburg und ihrer Lage zum Sihltal lassen sich die würmeiszeitlichen Eintiefungsbeträge einigermaßen abschätzen. Noch im Zürich-Stadium funktionierte die zur Sihl abfallende Talung Hinterbach-Sarbach-Tal-Schiffli als seitliche Schmelzwasserrinne des durch das Zugerseetal abfließenden Armes des Muota/Reußgletschers. Der Ägerilappen reichte in diesem Zeitpunkt nur noch bis Unterägeri. An der Einmündung ins Sihltal erkennen wir zwischen Tal und Schiffli eine Eintiefung des Sihltales um rund $50 \mathrm{~m}$.

Nach dem Schlieren-Stadium dürfte auch das Sihltal zwischen Sihlbrugg und Adliswil kräftig ausgeräumt worden sein. Ob dabei - etwa bei der rund $70 \mathrm{~m}$ betragenden Eintiefung von Langnau am Albis - eine präexistente Rinne, die während der Hochwürmeiszeit von Schutt und Eis erfüllt war, wieder ausgeräumt wurde, oder ob diese Eintiefung längs einer Bruchzone in die Molasse erst damals erfolgte, entzieht sich noch unserer Kenntnis.

Hingegen muß die postglaziale Tiefenerosion im Sihltal recht gering gewesen sein, da die Sihl, seitdem sich der Eislappen des von Baar gegen Sihlbrugg vorstoßenden Muota/Reußgletschers wieder zurückzog, kaum merklich eingeschnitten hat, liegt doch der Sattel zwischen Baar und Sihlbrugg nur etwa $12 \mathrm{~m}$ höher als das Sihlbett bei Sihlbrugg. Sehr wahrscheinlich war im Postglazial die Erosionsbasis bereits sehr früh erreicht.

Am nördlichen Abfall der Schotterplatte der Baarburg und des Josefsgütschs stellen sich neben Wallmoränen auch größere Sackungen ein, was wohl auf eine Unterschneidung durch Schmelzwässer zurückzuführen ist, die beim Abschmelzen des Eises ihres Gegendruckes beraubt worden waren und niederbrachen.

\section{DIE SCHOTTER ZWISCHEN ZUGERSEE UND DEM KNONAUER AMT}

Die Schotter des Knonauer Amtes gaben neulich zu einer kleinen Kontroverse Anlaß. H. Suter (1960: 501) erkannte bei geologisch-technischen Studien, die früher «z. 'T. als lokale Schwemmschotter der letzten Vergletscherung» aufgefaßten Schottervorkommen als einen zusammengehörigen Schotterstrang. 
Da die Schotter von Würm-Moränen diskordant überlagert werden, bezeichnete sie H. SUTER als präwürmische Rinnenschotter, die er (S. 508) als «mit dem Rückzug und Zerfall der gewaltigen Eismassen der größten Eiszeit» in Zusammenhang brachte.

Von Würm-Moränen bedeckte Schotter brauchen jedoch nicht eine Eiszeit älter zu sein, sondern lediglich prähochwürmeiszeitlich. Hingegen dürfte die Rinne selbst im Spätriß angelegt worden sein. Auf Grund der flachen Lagerung und ihrer Lage zu den heutigen Talläufen und zu würmeiszeitlichen Gletscherrändern sowie auf Grund ihrer fluviatilen Natur sind die Schotter selbst als frühwürmeiszeitlich zu deuten.

Suter (1960) brachte sie einerseits mit den Schottern zwischen Reuß- und Bünztal, andererseits mit den Sihl- und Lorzenschottern in Zusammenhang, da sie neben vorherrschenden Kalk- und Dolomitgeröllen, roten und hellen Graniten, nicht seltenen Radiolariten und Grüngesteinen aus der subalpinen Molasse verschiedentlich wenig gerundete Verrucanogerölle, Nummulitenkalke und Kristallingerölle aus dem Bündner Oberland enthalten, die nur aus dem Linth/Rheinsystem stammen können und während der Riß-Eiszeit in den Grenzbereich von Linth/Rhein- und Muota/Reußgletscher verfrachtet worden sein müssen (cf. S. 216).

J. Kopp (1961b) präzisiert zunächst die Altersdeutung der Schotter des Knonauer Amtes als würmeiszeitliche Vorstoßschotter, wobei er sie allerdings altersmäßig mit den Schottern von Menzingen korreliert. Die Menzinger Schotter sind jedoch streng genommen etwas jünger - hochwürmeiszeitlich - da sie erst kurz vor und während des Würm-Maximums zur Ablagerung gelangen konnten (cf. S. 218).

Genetisch deutet Kopp die Schotter zwischen Zugersee und dem Knonauer Amt als Ablagerungen des «granitfreien Muota-Ägeriarmes des Reußgletschers».

Da zur Zeit, als die Sihl- und Lorzenschotter abgelagert wurden, die Ebene von Baar, das untere Lorzental und das Reußtal bis über Maschwanden heraus bereits vom vorstoßenden Zugersee-Arm des Muota/Reußgletschers erfüllt waren, konnte die Lorze - damals vor allem Schmelzwässer des über Sattel bis gegen Unterägeri vorrückenden Ägerilappens des Muota/Reußgletschers - nur dem nordöstlichen Eisrand des Zugersee-Armes folgen und, zusammen mit seitlichen Schmelzwässern dieses Eislappens, durch das Knonauer Amt abfließen, was sich mit dem Geröllinhalt der Schotter zwischen Zugersee und Knonauer Amt bestens deckt.

Sehr wahrscheinlich gelangten in diesem Zeitpunkt auch die «Deltaschotter» von Blickensdorf zur Ablagerung. J. Kopp (1961: 651) betrachtet diese vom Früeberg bis zum Zimbelwald ( $2 \mathrm{~km} \mathrm{~N}$ bzw. W Baar) verfolgbaren Schotter - wohl in Anlehnung an R. FREI (1912: 68) - als rißeiszeitlich. Da sie ebenfalls Verrucano (Sernifit)-Gerölle führen, stellenweise - besonders etwa in dem bei Blickensdorf von NW mündendem Tobel der Bachtalen - über eine Höhe von über $80 \mathrm{~m}$ aufgeschlossen sind und stets von Würm-Moränen des Muota/Reußgletschers überlagert werden, ist ein $\mathrm{Zu}$ sammenhang mit den Sihl- und Lorzenschottern nicht von der Hand zu weisen. Die verschiedensten Schüttungsrichtungen dürften auf einen Eisrand-Stauschotter hinweisen, wobei Wasser z. T. subglazial abfloß.

Die etwas höher gelegenen Schotter im Steinhuser Wald (2 km NE Steinhausen) und im Schönbüelwald ( $2 \mathrm{~km} \mathrm{~N}$ Baar) dürften abgelagert worden sein, als der Muota/Reußgletscher bereits etwas weiter vorgestoßen war und dadurch die randlichen Schmelzwässer immer stärker gegen die Albiskette hin abgedrängt wurden. Diese Deutung wird auch durch die von KOPP (1961 b: 650) angeführte Tatsache gestützt, daß sich die Schotter zwischen Zugersee und Knonauer Amt über eine breite Zone verfolgen lassen. Als NE-Begrenzung der Schotter im Knonauer Amt gibt Kopp eine Linie an, die von Deinikon (1,5 km NE Baar) über Hauptikon-Mettmenstetten bis Dachelsen (2 km SSW Affoltern am Albis) verläuft. 
Damit ergeben sich weitgehendste Parallelen zwischen dem eiszeitlichen Geschehen im Muota/Reußsystem und demjenigen im Linth/Rheinsystem, wo sich - etwa bei Wädenswil - ebenfalls zwei Schotterfluren einstellen: einerseits eine tiefere, die Eisrandschotter im Reitbachtal SE von Wädenswil und die Schotter der Halbinsel Au, andererseits eine höhere, dokumentiert durch die Vorkommen von Altschloß und beim Waisenhaus Wädenswil.

Den Schottern zwischen Zugersee und Knonauer Amt würden im Glattal die Aatalschotter entsprechen, die, genau wie die Schotterplatte im Knonauer Amt, von dem zum Maximalstand vorstoßenden würmeiszeitlichen Gletscher überfahren worden sind, wobei die Oberfläche ebenfalls in eine eindrucksvolle Drumlinlandschaft verwandelt wurde.

Die von H. Suter (1960) als alter Lorzenlauf gedeutete Rinne wird von J. Kopp (1961 b) mit einem alten, schon von früheren Autoren angenommenen, aus dem $\mathrm{Zu}-$ gersee ausfließenden Reußlauf in Zusammenhang gebracht. Auf Grund einer zwischen Küßnacht am Rigi und Immensee abgeteuften Bohrung (cf. S.214) sowie einer Grundwasserbohrung, die 1,5 km ENE Cham niedergebracht wurde und die unter postglazialen Alluvionen der Lorze in $54 \mathrm{~m}$ Tiefe $(=365 \mathrm{~m})$ in mächtigen Seetonen verblieb, hält KopP diesen alten Reußlauf für erwiesen.

Analoge Verhältnisse wie bei Cham konnte Kopp bei der Bohrung Ochsenbach zwischen Baar und Zug feststellen. Solche Seetone sind jedoch in Übereinstimmung mit Bohrungen im Limmat- und im Glattal (in R. HANTKE 1959a) wohl am ehesten als riß/würm-interglaziale Seeablagerungen zu deuten, da sie sich im Limmattal auch außerhalb des Maximalstandes des würmeiszeitlichen Linth/Rheingletschers unter Schotterablagerungen finden. Damit ergäbe sich für die von SutrR postulierte Rinne im Knonauer Amt wiederum ein spätrißeiszeitliches Alter (cf. S. 220). Die in 440$450 \mathrm{~m}$ gelegenen Schotter der Kiesgrube an der zugerisch-zürcherischen Grenze zwischen Cham und Knonau (3,5 km N Cham), sowie die vom würmeiszeitlichen Reußgletscher überprägten Schotter im Raum Maschwanden-Obfelden-Ottenbach liegen aber eindeutig höher, sie sind somit sicher jünger, frühwürmeiszeitlich.

Die tiefer gelegenen, sandreichen Schotter von Maschwanden und von Mülibach (zwischen Obfelden und Ottenbach), die sich durch deutliche Schrägrichtung und durch eine in $404 \mathrm{~m}$ gelegene Übergußschicht auszuzeichnen, werden von J. KopP (1961b: 652) als nach dem Rückzug des Reußgletschers aus dem Zungenbecken von Bremgarten in den spätwürmeiszeitlichen Bremgartensee geschüttete Deltas des Haselund des Lindenbaches betrachtet. Die beiden Deltas würden somit die Akkumulationsprodukte darstellen zu den in die frühwürmeiszeitlichen Vorstoßschotter und stellenweise bis in die sandige Molasse eingeschnittenen Hohlformen der Talung HäglimoosHaselbach und des Lindenbaches. Der Geröllinhalt wäre praktisch identisch mit demjenigen des frühwürmeiszeitlichen Vorstoßschotter, da es sich dabei nur um eine spätere Umlagerung handeln würde; die etwas sandreichere Fazies wäre der ausgeräumten, sehr weichen Molasse zuzuschreiben.

In der Tat läßt sich auch die NE Blickensdorf in $490 \mathrm{~m}$ einsetzende Talung des Häglimooses am zwangslosesten als spätwürmeiszeitliche Abflußrinne deuten. Gegen ein jüngeres, postglaziales Alter spricht vor allem das heute fehlende Gewässer.

\section{ZUSAMMENFASSUNG}

Während früher sowohl im Grenzgebiet zwischen Linth/Rhein- und Muota/Reuß-System als auch in jenem zwischen Linth/Rhein- und Bodensee/Rheingletscher zahlreiche, gegenüber den heutigen Talsystemen recht hoch gelegene Schottervorkommen für präwürmeiszeitlich galten teilweise wurden sie sogar als günzeiszeitliche Deckenschotter aufgefaßt — fügen sich die meisten dieser Vorkommen zwangslos als beim Vorstoß der würmeiszeitlichen Gletscher in seitli- 
chen Abflußrinnen abgelagerte Schotter ins jungquartäre Geschehen ein. Damit ergeben sich auch Abschätzungsmöglichkeiten für jungquartäre Eintiefungen, die besonders in Zeiten erhöhten Wasseranfalles durch Schmelzwässer erfolgten.

Da das Einsetzen der Schotterfluren stets an ganz bestimmte Eisrandlagen gebunden zu sein scheint, dürften sich damit im Linth/Rhein wie im Muota/Reuß-System mehrere, sich entsprechende Vorstoßstadien abzeichnen.

Durch das Vorrücken der Gletscher wurden frühere Schmelzwasserläufe sukzessive eingedeckt, so daß die Entwässerungsrinnen immer stärker seitlich abgedrängt wurden.

Früher als interglaziale Flußabschnitte interpretierte Rinnen konnten als spätwürmeiszeitliche Abflußrinnen erkannt werden, da in der letzten Interglazialzeit im Zürichseegebiet und in den Talsystemen des Vierwaldstättersees, des Lauerzer- und des Zugersees die heutigen Talsohlen bereits durch die rißeiszeitlichen Gletscher geschaffen waren und in der letzten $Z_{\text {wi- }}$ scheneiszeit von ausgedehnten Seen eingenommen wurden, womit in diesen Abschnitten Tiefenund Seitenerosion weitgehend unterbunden waren.

Über ältere Talläufe sind wir sowohl im Reuß- als auch im Linthsystem immer noch viel zu sehr auf Mutmassungen angewiesen, da schlüssige Beweise noch kaum erbracht worden sind.

\section{LITERATURVERZEICHNIS}

Aeppli, A. (1894): Erosionsterrassen und Glazialschotter in ihrer Beziehung zur Entstehung des Zürichsees. Beitr. geol. Karte Schweiz [NF] 4. - (1904): Aus der Geologie des Kantons Zug. Zuger Neujbl. 1904. ANDREsen, H. (1961): Beiträge zur Geomorphologie des östlichen Hörnli-Berglandes. Im Druck. Brückner, E. (1909): in Penck, A. und Brückner, E.: Die Alpen im Eiszeitalter, 2. Jena (Tauchnitz). FREI, R. (1912): Monographie des schweizerischen Deckenschotters. Beitr. geol. Karte Schweiz, [NF] 37. - (1914): Geologische Untersuchungen zwischen Sempachersee und Oberem Zürichsee. Beitr. geol. Karte Schweiz, $[\mathrm{NF}]$ 45/1. FREY, O. (1907): Talbildung und glaziale Ablagerungen zwischen Emme und Reuß. Neue Denkschr. schweiz. naturf. Ges., 4I. FRÜH, J. (1907): Zur Morphologie von Brunnen-Schwyz. Eclogae geol. Helv., g/3: 399-407. Gassmann, F. (1961): Schweremessungen in der Umgebung von Zürich. Beitr. Geologie Schweiz, Geophys., 3. HaNtKe, R. (1958): Die Gletscherstände des Reuß- und Linthsystems zur ausgehenden Würm-Eiszeit. Eclogae geol. Helv., 5 r /1: 119-149. - (1959a): Zur Altersfrage der Mittelterrassenschotter. Die riß/würminterglazialen Bildungen im Linth/Rheinsystem und ihre Äquivalente im Aare/Rhone-System. Vjschr. naturf. Ges. Zürich, ro4/1: 1-47. - (1959b): Zur Phasenfolge der Hochwürmeiszeit des Linth- und des Reußsystems, verglichen mit derjenigen des Inn- und Salzachsystems sowie mit der nordeuropäischen Vereisung. Vjschr. naturf. Ges. Zürich, ro4/Schlußh.: 390-402. - (1960): Zur Gliederung des Jungpleistozäns im Grenzbereich von Linth- und Rheinsystem. Geographica Helvetica, ${ }_{5} / 4$ : 239-248. - (1961a): Tektonik der helvetischen Kalkalpen zwischen Obwalden und dem St. Galler Rheintal, Vjschr. naturf. Ges. Zürich, Io6/1: 1-212 (Habilitationsschrift). - (1961b): Die Nordostschweiz zur Würm-Eiszeit. Eclogae geol. Helv., 54/1: 123-132. - (1962): cf. Suter, H.l-. Heim, A Lв. (1891): Die Geschichte des Zürichsees. Neujbl. natf. Ges. Zürich. - (1894a): Die Entstehung der alpinen Randseen. Vjschr. naturf. Ges. Zürich, 39/1: 66-84. - (1894b): Die Geologie der Umgebung von Zürich. Compte Rendu de la 6e session du Congrès géol. internat. à Zurich. Lausanne. - (1919): Geologie der Schweiz I. Leipzig (Tauchnitz). JäCKLı. H. (1956): Talgeschichtliche Probleme im aargauischen Reußtal. Geographica Helvetica, $I_{I} / 1$ : 46-59. KLeiber, K. (1938): Geologische Untersuchungen im Gebiet der Hohen Rone. Eclogae geol. Helv., 3o/2: 419-430 (1937). Kopp, J. (1937): Die Bergstürze des Roßberges. Eclogae geol. Helv., 2q/2: 490-493 (1936). - (1945): Erläuterungen zum Geologischen Atlas der Schweiz, 1:25 000, Bl. 186-189. Beromünster-Eschenbach. Geol. Komm. - (1961a): Alte Flußläufe der Muota und der Steiner Aa zwischen Rigi und Roßberg. Eclogae geol. Helv., 53/2: 517-519 (1960). - (1961b): Zur Diluvialgeologie des Gebietes zwischen Zugersee und Knonauer Amt. Eclogae geol. Helv., 53/2: 648-653. PavoN, N. (1953): Die rückläufigen Terrassen am Zürichsee und ihre Beziehungen zur Geologie der Molasse. Geographica Helvetica, 8/3: 217-226. - (1955): Molassetektonik, Terrassen und Schotter zwischen Glattal, oberem Zürichsee und Sibltal. Verhandl. schweiz. naturf. Ges. 135, Porrentruy. - (1957): Geologie der Zürcher Molasse zwischen Albiskamm und Pfannenstiel. Vjschr. naturf. Ges. Zürich, roz/5: 117-315. SPECK, J. (1946): Exkursion No. 8: Zug-Lorzentobel-Schönegg. In: Geologische Exkursionen in der Umgebung von Zürich. Geol. Ges. Zürich. Zürich (Leemann). - (1953): Geröllstudien in der subalpinen Molasse am Zugersee und Versuch einer paläontologischen Auswertung. Zug (Kalt-Zehnder). STAUB, R. (1939): Prinzipielles zur Entstehung der alpinen Randseen. Eclogae geol. Helv., ${ } I / 2$ : 239-258. SUTER, H. (1944): Glazialgeologische Studien im Gebiet zwischen Limmat, Glatt und Rhein. Eclogae geol. Helv., 37/1: 83-97. - (1956): Geologie des Sihltales. Bl. Vereinig. Pro Sihltal, 6. - (1960): Beitrag zur Diluvialgeologie des Knonauer Amtes, Kanton Zürich. Eclogae geol. Helv., 52/2: 499-509. - / HANTKE, R. (1962): Geologie des Kantons Zürich mit Grenzgebieten. Zürich (Leemann). Landeskarte der Schweiz. 1:25 000: LK 1111, 1112, $1131,1132,1151$ und 1152. 


\section{PROBLEMES QUATERNAIRES ENTRE LES DEUX SYSTEMES MUOTA/REUSS ET LINTH/RHIN}

Une révision des graviers dans les deux systèmes glaciaires du Linth/Rhin et de la Muota/ Reuss, attribués autrefois à la haute terrasse, a montré que la plupart de ces cailloutis s'expliquent beaucoup mieux comme graviers déposés dans des chenaux d'eau de fonte marginales lors de l'avancée des glaciers wurmiens (Frühwürm). Le fait que ces cailloutis font leur apparition à des endroits bien déterminés par rapport aux bords isochrones des glaciers fait admettre l'existence, pendant la glaciation wurmienne, non seulement de stades de retraite, mais aussi de stades d'avancement. Lors de l'avancée successive, mais discontinue, des glaciers wurmiens, les anciens chenaux des eaux de fonte ont été envahis par la glace, de sorte que les eaux de fonte étaient forcées de trouver d'autres cours, situées plus haut par rapport aux fonds des vallées qui se remplissaient de plus en plus avec de la glace vers le maximum de la glaciation (Hochwürm). Des torsos recouverts de dépôts fluviatiles plus jeunes, interprêtés autrefois comme cours d'eau interglaciaires, ont été reconnus comme d'âge wurmien tardif (Spätwürm).

\section{DIE PROVENCE ALS KULTURLANDSCHAFT}

\section{Willy Meyer}

\section{Mit Farbtafel}

Bedarf es eines Beweises dafür, daß man die Begriffe «Landschaft» und «Kultur» zu einem einzigen verschmelzen dürfe? Die Provence bietet einen solchen Beweis aufs evidenteste: Erde, Mensch, Tätigkeit - und was Kunst und Kultur in ihr, aus ihr gemacht haben, gehört ganz eng zusammen. Man lese den schönen Roman, den Marie Mauron «Le Royaume errant» betitelt hat: wie sie die zwei Typen des scholleverbundenen, seßhaften Bauern und des die Transhumances geleitenden, wandernden Hirten vor uns hinstellt und ineinander übergehen läßt, das ist eine wundervolle Einheit, nicht nur erdichtet etwa, sondern der Wirklichkeit entnommen, vom Charakter des Landes geprägt und zugleich es prägend. Gewiß, nicht jeder Landschaft eignet eine so spezifische Kultur; deshalb wird man nicht jede als Kulturlandschaft bezeichnen dürfen.

Man denke aus der rhodanischen Provence ihre Römerbauten fort - sie wird sich bis zur Unkenntlichkeit verändern; doch auch diese Monumente werden eines Wesentlichen entbehren: ihres Reliefs, ihres Rahmens. Wo anders möchten zwei der kleineren von ihnen, das Kenotaph der Julierprinzen und der ihm benachbarte Stadtbogen des kelto-römischen Glanum, so einmalig zu wirken wie auf ihrem Hügel der «Antiques», im Angesichte der Alpilles!

Und fällt der Blick von dieser Höhe auf die nahe romanische Klosterkirche SaintPaul-de-Mausole: mußte nicht Van Gogh, der dort zwei schwerste Lebensjahre zubrachte, Licht, Form und Farbe der Provence entdecken, um - er selbst zu werden und sich zu erfüllen?

In der Tat, es ist eine Magie um diese Kulturlandschaft der Provence, etwas von der Berührung mit einem Zauberstab, aus der ein Geben und Nehmen wird, ein durchaus persönlicher Reiz, eine Fülle, ja Überfülle an Bildern, ob sie nun aus Mythen und Legenden, druidischen Erinnerungen, Heiligengeschichten, oder aus historischem Geschehen aufsteigen.

$\mathrm{Da}$ ist das «Adlernest» der Baux, noch als Ruine Inbegriff der Burgen und Schlösser, die der Feudalismus errichtete, einer der Liebeshöfe, von deren einem zum andern die Troubadoure zogen. Ihr Weg war nicht immer nur Poesie. Manchen traf furchtbar die Eifersucht des Schloßherrn, dessen Gattin er preisend besang: auch Leidenschaft der Rache gehört zur Vielfalt einer Landschaft, die nicht nur unter ihrer südlichen Sonne erglüht, sondern tagelang von der Gewalt des Mistralwindes gepeitscht wird, bis er, der «Mangefange», der Schlammfresser, das letzte $\mathrm{Naß}$ ihrer 